\title{
Le loup en tribunes : analyse comparée de deux discours argumentatifs sur une espèce controversée
}

\author{
Marie Chandelier ${ }^{1}$, Raphaël Mathevet ${ }^{2}$, Agnès Steuckardt ${ }^{1}$, Jean-Marc Sarale ${ }^{1}$ \\ 1 Linguiste, Université Paul-Valéry-Montpellier 3, UMR5267 Praxiling, 34090 Montpellier, France \\ 2 Écologue et géographe, CNRS, UMR5175 Centre d'écologie fonctionnelle et évolutive, 34293 Montpellier Cedex 5, France
}

Pour NSS, les controverses, ici sur la biodiversité, sont un moment nécessaire du travail interdisciplinaire. Cet article mobilise une méthode scientifique, l'analyse argumentative, pour décrypter, de manière neutre quant au fond, ce qui oppose les partisans et les opposants au maintien d'une population sauvage. Il révèle à quel point conceptions scientifiques et représentations socialement situées se mêlent pour apprécier une situation. La revue partage le point de vue des auteurs qui font le pari qu'une telle analyse peut faciliter le dialogue entre les protagonistes.

La Rédaction

\section{Mots-clés :} environnement ; agriculture ; biodiversité ; sciences de la conservation ; linguistique
Résumé - Cet article propose une approche discursive de la controverse liée à la présence du loup en France. L'analyse comparée de deux plaidoyers, l'un défavorable, l'autre favorable au retour de l'espèce, parus respectivement dans Libération et Le Monde fin 2014, met en évidence l'existence de représentations opposées de l'équilibre environnemental. Les groupes de signataires privilégient un équilibre entre l'élevage et la nature, perturbé par le loup (Libération), et un équilibre entre le loup et la nature, perturbé par l'homme (Le Monde). La communauté socioécologique décrite doit sa stabilité à la mise à l'écart totale ou partielle d'un acteur du territoire. Les marques de présence des acteurs mettent en évidence deux autorités aboutissant à la formation d'un discours objectivé par un ethos scientifique (Libération), et d'un discours subjectivé par un ethos militant (Le Monde).
Keywords: environment; agriculture; biodiversity; conservation sciences; linguistics

\begin{abstract}
The wolf in opinion pages: a comparative analysis of argumentative discourses on a controversial species. The recovery of the grey wolf population in France has generated numerous conflicts, which have been studied by many disciplines including the social sciences. Our paper shows that a discursive approach to these controversies can offer new insights and help to understand the continuing oppositions between the various actors involved. A comparative analysis of the argumentative discourses developed in two articles published by two national French newspapers, Libération and Le Monde, highlights two contrasting representations of environmental equilibria. The first aims at an equilibrium between livestock and nature (Libération), the second at an equilibrium between wolves and nature (Le Monde), each equilibrium being disrupted respectively by wolves and humans. The stability of the social-ecological community is based on the exclusion of one actor. Despite a similar formal structure, each article develops a specific authority through the interaction between the peritext and the text, and through the enunciative system in order to legitimate the author's representations. In the Liberation article, we show that the scientific authority serves to objectify and thus legitimate the argumentative discourse, whereas in Le Monde, a militant authority subjectifies the discourse. In both papers, the authority is also closely linked to the identity of the country (France). Both articles have a polemical dimension, which is however more explicit in Le Monde where the opponents are clearly identified.
\end{abstract}

Auteur correspondant : M. Chandelier, marie.chandelier@cefe.cnrs.fr 
En Europe et en France, le retour du loup gris (Canis lupus) donne lieu à des conflits virulents au sein et en dehors des espaces naturels protégés. Les controverses et les stratégies de politiques publiques élaborées depuis le retour de l'espèce témoignent des difficultés de l'homme à s'insérer dans une communauté socioécologique rassemblant dans un même collectif humains et non-humains (Latour, 1999). Les controverses que la présence de l'espèce engendre ont fait l'objet d'études historiques (Moriceau, 2011), ethnosociologiques (Lescureux et Linnell, 2013), géographiques (Benhammou, 2007), sociologiques (Mounet, 2007), écologiques et biologiques, ou encore éthologiques (Landry, 2006). Les travaux menés en sciences sociales, pour la plupart de terrain, sont ancrés spatialement (Benhammou, 2007 ; Mauz, 2006 ; Mounet, 2007) et montrent que le loup est une entité autour de laquelle les acteurs se positionnent et s'affirment (Doré, 2011). Dans sa thèse, Antoine Doré propose de considérer les controverses au sujet de la présence du loup par le prisme de la sociologie de la traduction (Akrich et al., 2006) et des travaux de Jacob von Uexküll publiés en 1934 (voir l'édition traduite de 2004). Chaque acteur (humain et non humain) est envisagé selon les liens qu'il entretient avec les autres (humains et non humains). L'auteur entend ainsi dépasser les catégories construites autour de l'espèce " loup », alors même que les groupes impliqués dans la controverse peinent à considérer cette dernière comme un acteur à part entière.

Entreprendre une étude des conflits impliquant le loup par l'analyse du discours donne la possibilité d'accéder à la mise en scène des positions des individus interagissant plus ou moins directement avec le loup.

La diffusion de la question du retour et de l'expansion du loup vers des publics élargis (Doré, 2011) a notamment été permise par les médias (régionaux et nationaux). Les articles de presse consacrés au retour du loup ont été étudiés en communication (Lits, 2008). La recherche de Marc Lits se focalise sur le réinvestissement des mythes et des récits toujours présents dans la mémoire collective. Mais ces analyses ne traitent pas du discours argumentatif dans la controverse, ni de la place attribuée aux différents acteurs dans les médias.

Dans les débats au sujet de la présence du loup en France, les discours produits par les groupes impliqués sont pour la plupart argumentatifs. L'intérêt d'une analyse de ces discours est de déconstruire les arguments utilisés, afin d'identifier leur structure (Plantin, 1990 ; Toulmin, 1958) et de connaître les mécanismes prédominants de l'argumentation en fonction des positions défendues et des acteurs qui les défendent (Perelman et Olbrechts-Tyteca, 1958). Une meilleure connaissance de ces mécanismes permet d'accéder aux représentations qui sous-tendent chacune des revendications, et aux relations qui unissent ou divisent les personnes impliquées dans les débats. À la différence des enquêtes de terrain menées en sociologie, l'analyse du discours s'intéresse ici à des textes déjà produits, dont les contraintes d'écriture ne dépendent pas de la place du chercheur lors de l'entretien. Le chercheur n'est pas confronté au paradoxe de l'observateur (Labov, 1972) ; c'est-à-dire qu'il n'est pas susceptible d'influencer, par sa seule présence, les données à étudier. L'analyse du discours peut contribuer à traiter à la fois des relations homme/nature, des interactions domestique/sauvage dans le temps et l'espace ou encore du pilotage de la biodiversité (Blandin, 2009) et de nos solidarités avec les humains au sujet de la biodiversité, tout comme de nos solidarités avec les non-humains (Mathevet et al., 2010 ; Mathevet, 2012). Nous souhaitons mettre en évidence ce que l'analyse du discours peut apporter aux sciences de la conservation, à travers une analyse comparée de deux articles, « Loups : plaidoyer pour des écosystèmes non désertés par les bergers » (Texte A) et « Cessons de diaboliser le loup! » (Texte B), respectivement parus en octobre et décembre 2014 dans Libération et Le Monde ${ }^{1}$.

Le corpus met en perspective deux argumentations construites par deux groupes de scientifiques. Bien qu'il $\mathrm{s}^{\prime}$ agisse de groupes d'acteurs restreints, ces derniers incarnent des positions circulant plus largement dans les débats : pour défendre une vision spécifique de la protection de la nature, les auteurs reprennent et réinvestissent des données scientifiques mais aussi des données utilisées par d'autres groupes d'acteurs (agriculteurs, écologistes, scientifiques, politiques...). La remobilisation de ces données dans les commentaires en ligne suscités par chaque article ( 54 sur le texte A et 64 sur le texte $\mathrm{B}$ ) montre que les textes $\mathrm{A}$ et $\mathrm{B}$ cristallisent une polarisation de la controverse.

L'autorité scientifique est susceptible d'apporter une légitimité à l'argumentation; aussi ce corpus permet-il d'interroger les rapports qu'entretiennent les discours scientifiques et les discours militants dans la gestion des politiques liées à la nature.

«Cessons de diaboliser le loup ! » est une réponse au « Plaidoyer ». Chacun de ces deux articles défend auprès d'un public élargi - le lectorat des quotidiens nationaux - une position respectivement favorable et défavorable à la présence du loup sur le territoire français. Les signataires, pour la plupart scientifiques, dressent un bilan de la place du loup et du pastoralisme en France. La structure des arguments, ainsi que les procédés rhétoriques et stylistiques utilisés aboutissent à la confrontation de deux discours qui s'opposent, alors même qu'ils

\footnotetext{
1 Cette étude prend place dans le cadre d'une thèse de doctorat intitulée Le loup et le sanglier dans la presse française contemporaine. Analyse comparée des discours argumentatifs sur deux espèces controversées. Ce travail est coencadré par Agnès Steuckardt (Praxiling) et Raphaël Mathevet (équipe « Dynamique des systèmes socio-écologiques » [DSSE] au Centre d'écologie fonctionnelle et évolutive [CEFE]).
} 
sont construits à partir de données proches, puisées dans la structure du réel, c'est-à-dire dans les jugements admis et circulants (Tab.).

L'analyse comparée des articles permet d'identifier les données argumentatives retenues par les énonciateurs dans les textes $\mathrm{A}$ et $\mathrm{B}$, et de saisir à quel stade de l'argumentation la divergence des points de vue s'opère. Pour cela, nous nous intéresserons ici à la mise en scène, par les signataires des deux tribunes, des interactions entre les acteurs de la controverse en fonction de leur ancrage à la fois temporel et spatial sur le territoire. Nous identifierons le rôle que joue le statut des signataires dans la construction du discours argumentatif, en analysant les marques de présence, dans les articles, des auteurs, de leurs soutiens, ainsi que des groupes d'acteurs auxquels ils s'opposent.

\section{L'inscription des acteurs dans le temps : perspective comparée}

Dans la phase de cadrage ${ }^{2}$ du texte $A$, les « paysages emblématiques de montagnes, collines, bocages et marais » sont envisagés selon l'impact positif que les activités pastorales ont sur eux. Les auteurs livrent au lecteur une définition orientée des paysages qui privilégie, dans leur constitution, le facteur humain : "Ils se dégradent rapidement quand ils ne sont plus entretenus par le pâturage des troupeaux notamment ». En se focalisant sur les objets de discours « loup », « troupeaux » et " pratiques paysannes ", le cadrage place en opposition discursive deux agents animés non humains (loup, troupeaux), et produit un effacement des agents animés humains (bergers, éleveurs), bien que ces derniers soient à l'origine des pratiques pastorales. Cela conduit à une focalisation sur les pratiques agropastorales plutôt que sur les bergers et les éleveurs qui les développent. Les milieux ouverts dont il est question ici acquièrent une légitimité par l'inscription dans la durée de l'équilibre des liens entre l'activité pastorale et l'environnement : «Nos paysages [...] sont constitués d'une mosaïque de milieux façonnés au fil des siècles par les pratiques paysannes ". Le caractère ancien et durable de cet équilibre, explicité par l'expression " au fil des siècles ", contribue à donner au loup le rôle de perturbateur des relations homme-environnement : «Or, en de nombreuses régions, les troupeaux subissent l'assaut des loups ».

Dans le texte B, le cadrage oppose deux objets de discours : « les éleveurs » et " le loup », et attribue la dégradation de la biodiversité aux éleveurs. La relation de

2 La phase de cadrage de l'argumentation permet de construire un lieu de consensus entre l'énonciateur et l'énonciataire. Elle est la base de l'argumentation à venir (Breton, 2009). concurrence qui s'instaure entre ces deux objets de discours conduit à considérer l'homme en perturbateur d'un équilibre écologique existant « depuis des milliers d'années ». La chronologie retraçant la présence de l'espèce sur le territoire donne au loup une implantation antérieure à celle du pastoralisme. Elle met en tension les causes de la disparition de l'espèce et celles de son retour en France. L'usage de l'adverbe « naturellement » ( « revenus naturellement en France en 1992 ») est interdiscursif $^{3}$; il renvoie, en les infirmant, aux thèses polémiques mettant en doute le retour naturel du loup. Le contraste établi entre les causes naturelles de la présence de l'espèce et la cause humaine de sa disparition tend à attribuer une plus forte légitimité à l'occupation actuelle du territoire par le loup. Dans le texte A, l'année 1992 est évoquée pour dater non pas le retour du loup, mais la création de « directives européennes [dont le but est de] promouvoir la gestion des milieux agropastoraux ». L'association de cette date à la mise en place de mesures agropastorales, alors même qu'elle est usuellement liée au retour du loup en France, conduit le lecteur à envisager une incompatibilité entre la présence du loup, décrite comme une menace pour le pastoralisme dans le paragraphe 2, et les entreprises européennes de valorisation de l'agropastoralisme, qui ont lieu au même moment.

Les repères temporels des deux articles contribuent à positionner les acteurs de la controverse et les activités qui y sont impliquées. Dans le texte A, l'agropastoralisme entretient des liens de complémentarité anciens avec la biodiversité, que la présence du loup menace de défaire, alors que dans le texte $B$, le loup, dont la présence est antérieure à celle des activités pastorales, est menacé par l'homme. Les complémentarités et les ruptures présentées dans les articles font apparaître un point commun, la rupture d'un équilibre préexistant, mais deux causes opposées, respectivement en raison de l'action du loup (pour le texte A), et de l'homme (pour le texte B).

\section{L'inscription des acteurs dans l'espace : perspective comparée}

La hiérarchisation des acteurs du territoire à travers leur positionnement dans le temps et en fonction de l'impact sur un équilibre qui préexistait est renforcée par la description des relations qu'ils entretiennent dans l'espace. Les mêmes données argumentatives, dans chacun des documents, contribuent à forger des conclusions opposées.

\footnotetext{
3 Élaborée par Michel Pêcheux en 1975 (Maldidier, 1993), la notion d'interdiscours suppose que tout énoncé renvoie à un espace discursif constitué des discours déjà produits, avec lesquels l'énoncé entretient des relations influencées par des paramètres sociaux et historiques.
} 
Tableau. Analyse comparée des thèses et arguments des deux articles.

\begin{abstract}
TEXTE A. « Loups : plaidoyer pour des écosystèmes non désertés par les bergers » (Libération)
\end{abstract}

http:/ / www.liberation.fr/terre/2014/10/12/plaidoyer-pourdes-ecosystemes-non-desertes-par-les-bergers_1120258

\section{Position argumentée de la thèse}

\section{Thèse $\mathrm{A}$ : les loups sont dangereux}

\section{Paragraphe 1}

Phase de cadrage : définition orientée des paysages

emblématiques (situe le pastoralisme dans la durée, dans une relation de complémentarité avec le paysage)

Donnée : «Or, en de nombreuses régions, les troupeaux

subissent l'assaut des loups. »

Conclusion : "La gravité de la situation plaide pour l'adoption

de mesures d'urgence, sur le terrain comme dans le domaine

réglementaire. »

Loi d'inférence ${ }^{*}$ : conséquences négatives

Question argumentative du nombre

\section{Argument : les loups sont trop nombreux}

\section{Paragraphe 4}

Donnée 1 : «Installés partout dans les Alpes, les loups ont désormais gagné le Jura, [...] les plaines lorraines et champenoises. »

Donnée 2 : « Les décomptes officiels, en 2014, font état de vingt-sept meutes, dont les deux-tiers dans les Alpes du Sud. La population est de 300 loups adultes dans plus d'une vingtaine de départements, avec une croissance de $20 \%$ par an. »

Donnée 3 : « Chaque année, les pertes officielles font état de vingt à vingt-cinq brebis ou chèvres tuées en moyenne par loup adulte. »

Donnée 4: «Les attaques s'étendent aux génisses, veaux et chevaux. »

Donnée 5: «Elles se déroulent en alpages, mais aussi sur des landes et coteaux en vallées, en sous-bois, et jusque dans des prés. »

Conclusion : "L'enjeu est devenu national. »

Loi d'inférence : lieu commun de quantité (population de loups, nombre de proies, nombre d'espèces prises comme proies, variété des milieux naturels)
TEXTE B. « Cessons de diaboliser le loup ! » (Le Monde)

http:/ /www.lemonde.fr/idees/article/2014/12/14/cessonsde-diaboliser-le-loup_4540319_3232.html

\section{Thèse $B$ : les loups sont en danger}

Paragraphe 1

Phase de cadrage : implantation du loup sur le territoire antérieure à celle du pastoralisme

Donnée : «Tel syndicaliste paysan offre 1000 euros de prime pour la peau d'un animal, tel maire de village promet 2000 euros pour la capture d'un individu. Même les spécialistes s'y mettent!»

Conclusion : « Rien ne va plus pour les loups [...], ils redeviennent chaque jour un peu plus des cibles. » Loi d'inférence : exemples

\section{Contre-argument : les loups sont peu nombreux}

Paragraphe 6

Donnée 1 : « Les loups ne sont que 300 à 350 dans une vingtaine de départements, ce qui est peu au regard des densités des deux derniers siècles : 6500 animaux estimés au XVIII ${ }^{\mathrm{e}}$ siècle. »

Donnée 2: «Pourquoi la France ne pourrait-elle pas héberger quelques centaines de loups quand nos voisins, l'Espagne et

$1^{\prime}$ Italie, en tolèrent chacun 4 à 8 fois plus, sans avoir pour autant sacrifié le pastoralisme?»

Conclusion : "On tente donc une nouvelle fois de diaboliser le loup. »

Loi d'inférence : proportionnalité

Question argumentative de la régulation des écosystèmes par le loup

Argument : les loups ne jouent pas un rôle régulateur

\section{Paragraphe 2}

Donnée 1: «Dans le Grand Nord américain comme eurasien, ils sont considérés comme "espèce clé de voûte" des écosystèmes, indicatrice d'une nature redevenue ou restée sauvage. » Donnée 2 : « les loups [...] s'attaquent fréquemment aux troupeaux d'élevage en parfaite santé. »

Loi d'inférence : distinguo : le loup, espèce protégée, est, d'une part, considéré comme le représentant $\mathrm{d}^{\prime}$ « une nature redevenue ou restée sauvage ", au comportement régulateur de la vie sauvage, et, d'autre part, comme une menace au pastoralisme, présenté comme «l'une des agricultures les plus respectueuses de la biodiversité, reconnue [...] comme productrice d'une variété de services écosystémiques. »

* Dans le modèle développé par Toulmin, la donnée est l'élément qui est utilisé pour défendre la conclusion. La loi d'inférence est la proposition qui permet d'établir un lien de cohérence entre la donnée et la conclusion.

\section{Contre-argument : les loups jouent un rôle régulateur}

Paragraphe 7

Question rhétorique : « Mais faut-il croire ceux qui pensent qu'il négligerait ses fonctions de régulateur d'animaux sauvages pour s'attaquer aux proies domestiques saines? » (argumentation adverse [thèse + argument])

Donnée 1: "le loup attaque autant les uns que les autres. " Donnée 2 : « les meutes structurées ont tendance à se nourrir de proies sauvages. »

Conclusion: Il ne faut pas « croire ceux qui pensent qu'il négligerait ses fonctions de régulateur»

Loi d'inférence : cause (d'attaques sur sauvage/domestique, proies sauvages) 
Tableau. Suite.

TEXTE A. « Loups : plaidoyer pour des écosystèmes non
désertés par les bergers » (Libération)
http:/ / www.liberation.fr/terre/2014/10/12/plaidoyer-pour-
des-ecosystemes-non-desertes-par-les-bergers_1120258

Question argumentative de la régulation des écosystèmes par les troupeaux

Argument : les troupeaux jouent un rôle régulateur

Argument additionnel : les troupeaux font eux-mêmes partie de la biodiversité

\section{Paragraphe 3}

Cellule argumentative 1 :

Donnée 1: "Nombre d'espèces remarquables y ont trouvé refuge : bartavelle, lagopède, bouquetin, gypaète. »

Donnée 2: «Les mosaïques de pelouses, landes et prés-bois, entretenues par le pâturage offrent et renouvellent toutes sortes d'aménités [...] que les éleveurs s'évertuent à conserver. »

Conclusion : Les milieux agropastoraux « ont résisté à la banalisation des paysages et à l'artificialisation par l'agriculture conventionnelle. »

Loi d'inférence: conséquence positive

Cellule argumentative 2 :

Donnée : Les éleveurs s'évertuent à conserver « les brebis raïoles, brigasques et mourerous, les chèvres du Rove et du Poitou. » Conclusion : La biodiversité favorisée par le pastoralisme " est également domestique. »

Loi d'inférence : conséquence positive régulateur

Paragraphe 1 jours. »

\section{Paragraphe 2} sinistré »
TEXTE B. «Cessons de diaboliser le loup!» (Le Monde)

http:/ / www.lemonde.fr/idees/article/2014/12/14/cessonsde-diaboliser-le-loup_4540319_3232.html

Donnée : « En moyenne, les troupeaux sont passés de 200 brebis au milieu du $X X^{\mathrm{e}}$ siècle à plus de 500, voire 1000 à 3000 de nos

Conclusion : Les pratiques du pastoralisme ont bien changé

Loi d'inférence : conséquences négatives

Question rhétorique: "Mais la nature domestiquée est-elle l'idéal de la biodiversité ? » (assertion négative correspondante = négation de la thèse adverse)

Donnée : Le loup conduit à la diversification d'un «patrimoine

Conclusion adverse : "Les éleveurs "passionnés, inspirés par le respect du vivant" sont opposés de manière naïve à la situation "intenable" créée par le loup, mal suprême. "

Réserve : «On oublie en route, très opportunément, les impacts négatifs occasionnés par le pastoralisme, dont les pratiques ont bien changé. »

\section{Paragraphe 3}

Donnée: "Que dire aussi de la perte en diversité des insectes pollinisateurs et autres invertébrés, des criquets aux sauterelles, qui en dépendent pour se nourrir et se reproduire, et des maladies (brucellose, kératoconjonctivite, piétin) transmises par les concentrations excessives d'ovins à la faune sauvage de chamois et bouquetins?»

Conclusion : Le pastoralisme a des impacts négatifs sur l'environnement (paragraphe 2)

Loi d'inférence : conséquences négatives

Dans le paragraphe $4 \mathrm{du}$ texte A, la mention par accumulation des lieux de présence du loup et des attaques attribuées à l'espèce vise à montrer que le loup, fortement présent sur le territoire, y est nuisible. Les noms et pronoms utilisés tout au long de l'article pour référer au loup sont essentiellement à la forme plurielle , et conduisent ainsi à envisager l'animal en meute. Ce n'est pas l'espèce qui est évoquée, ni un de ses représentants, mais un ensemble d'individus. Le substantif «loup », lorsqu'il est en position sujet, et le pronom de la sixième personne, lorsqu'il réfère aux loups, sont

4 Le substantif « loup » est employé treize fois (c'est le mot lexical le plus utilisé dans le texte). Il compte douze occurrences au pluriel, et une seule au singulier. La référence à l'animal se fait également par l'emploi, à cinq reprises, du pronom «ils". Nous n'observons en revanche aucune occurrence de ce pronom au singulier. majoritairement associés à des verbes d'action (respectivement quatre occurrences sur six et trois sur cinq) : « manifestent »; « menacent»; « ont désormais gagné »; «ont perçu »; « négligent »; « arrivent »; « attaquent ». Ces verbes, dont trois sont axiologiquement négatifs (« menacer», « négliger », « attaquer »), attribuent une intentionnalité aux actions du loup de la même façon que les adjectifs « opportuniste », « intelligent », « inventif ». Cependant le terme « opportuniste » est ambigu car polysémique ; il désigne, dans le discours scientifique, la capacité d'une espèce à s'adapter à des milieux variés. Dans le discours commun, il possède une dimension axiologique négative assez forte, qui est en revanche absente du discours scientifique. Le texte A, publié dans un journal grand public, ne s'adresse pas à un lectorat spécialisé. Dans ces conditions, le sens de l'adjectif que le lecteur sera amené à privilégier dépend du 
cotexte, c'est-à-dire de l'environnement textuel. La présence du nom " comportement " (" comportement opportuniste ») laisse penser qu'il s'agit davantage $\mathrm{d}^{\prime}$ une considération scientifique. La mention du statut scientifique des signataires de l'article renforce la thèse d'un usage spécialisé du terme. En revanche, la présence du verbe axiologiquement négatif "négliger » donne aux actions du loup un caractère prémédité, et renvoie davantage à la dséfinition du mot «opportuniste » dans l'usage courant. La catégorie mobilisée par l'adjectif se construit ici par l'association du sens scientifique et de celui de l'usage courant (Waltereit, 2003). Le caractère spécialisé de la définition donne une autorité au discours communiqué, alors même que la définition connue du grand public conduit à une évaluation négative et stigmatisante du loup.

Le loup semble engagé dans une entreprise de conquête du territoire. Dans le paragraphe 4 , l'emploi graduel des verbes d'action "installer », " gagner », " arriver » attribue tout d'abord une base au loup («Installés dans les Alpes»), puis une zone déjà conquise (« les loups ont désormais gagné le Jura »), et enfin des espaces - les plus nombreux - en acquisition (« ils arrivent en Ardèche, en Lozère, dans le Cantal et l'Aveyron, les plaines lorraines et champenoises »). Le loup est ainsi présenté comme une espèce envahissante, fortement implantée sur le territoire. La juxtaposition des animaux susceptibles d'être attaqués (« Les attaques s'étendent aux génisses, veaux et chevaux ») élargit la menace du loup non plus uniquement aux «brebis ou chèvres », mais à d'autres espèces élevées par l'homme. Le loup devient ainsi une menace pour l'ensemble de la biodiversité domestique, alors même que la requalification du pastoralisme, dans le paragraphe 3 , donne à cette activité un rôle majeur pour la conservation de la biodiversité à la fois sauvage et domestique. Cela conduit à la formation d'un argument comparatif implicite, où l'activité pastorale contribue mieux à la biodiversité que le loup. La description de la progression spatiale de l'espèce, associée à une mise en danger de la conservation de la nature, oppose le loup à la biodiversité. La redéfinition $\mathrm{du}$ rôle du pastoralisme établit en revanche des liens forts entre la biodiversité et l'activité d'élevage, toutes deux menacées par la présence d'un grand carnivore. De cette façon, le loup tend à être mis à l'écart de la biodiversité, qu'il menace.

Dans le texte $\mathrm{B}$, le rapport de l'animal à l'espace est construit en contrepoint de celui du texte A. L'utilisation des mêmes données argumentatives que dans le texte $\mathrm{A}$ (nombre de loups présents sur le territoire, nombre de départements où l'espèce est recensée) conduit ici à une vision encore fragile de l'implantation du loup en France. La comparaison de ces données avec des données à la fois spatiales ( « nos voisins, l'Espagne et l'Italie, en tolèrent chacun 4 à 8 fois plus ») et temporelles («ce qui est peu au regard des densités des deux derniers siècles ») minimise l'importance du nombre de loups en France et de leur répartition sur le territoire. La forme négative restrictive "ne... que » contribue à diminuer le poids donné au nombre de loups en France dans le texte $A^{5}$. L'espèce fait partie intégrante d'une biodiversité fragile, qu'il est nécessaire de conserver. Les noms et pronoms utilisés pour référer au loup sont, dans l'article, majoritairement au singulier ${ }^{6}$, opposant au loup envisagé en meute le loup comme représentant de l'espèce ${ }^{7}$. Les verbes d'action dont le substantif « loup » est sujet sont diversifiés d'un point de vue axiologique : « mange », « attaque », « bénéficie, » « joue un rôle », « se réinstalle », « négligerait », « habitent », « redeviennent ». Le verbe axiologiquement négatif « négliger » est employé au conditionnel lors d'une reformulation de la thèse adverse, réfutée par les auteurs de l'article ${ }^{8}$. Le préfixe " re ", employé à deux reprises ("se réinstaller ", « redevenir ») souligne la présence antérieure du loup sur le territoire et oppose ces deux verbes aux verbes « arriver », « avoir gagné » du texte A, qui positionnait le loup en conquérant de territoires déjà occupés par les activités pastorales. C'est le retour de l'animal sur des lieux qu'il a déjà occupés qui est souligné, dans le but de légitimer son implantation en France. Si, dans le texte $\mathrm{A}$, l'adjectif «opportuniste » était utilisé pour qualifier les comportements du loup, l'adverbe « opportunément » caractérise, dans le texte $B$, la stratégie argumentative des auteurs du texte A. Il fait écho à l'intentionnalité des comportements du loup dénoncés dans le texte $\mathrm{A}$, et est en cela interdiscursif. Ici, ce sont les signataires de l'article de Libération qui, de façon intentionnelle, ne mentionneraient pas les «impacts négatifs occasionnés par le pastoralisme ». L'adverbe " opportunément » ne présente pas la polysémie de l'adjectif «opportuniste» ; le parallèle avec le comportement opportuniste reproché au loup est implicite et se situe dans l'interdiscours.

Les bienfaits de l'activité pastorale sur l'environnement sont remis en cause par la comparaison de la taille

\footnotetext{
5 «Les loups ne sont que 300 à 350 dans une vingtaine de départements » (texte B) ; "La population est de 300 loups adultes dans plus d'une vingtaine de départements, avec une croissance de $20 \%$ par an » (texte A).

6 Le substantif «loup » est employé vingt fois (c'est le mot lexical le plus utilisé dans l'article). Nous relevons quinze occurrences au singulier et cinq au pluriel. Le pronom personnel apparaît, lorsqu'il réfère au loup, deux fois au singulier et deux fois au pluriel.

7 Cette opposition est observable dès la lecture du titre des articles, qui comportent une occurrence de « loup » au pluriel pour le texte A (uniquement pour la version internet), et au singulier pour le texte $B$.

8 «Mais faut-il croire ceux qui pensent qu'il négligerait ses fonctions de régulateur d'animaux sauvages pour s'attaquer aux proies domestiques? ».
} 
des troupeaux actuels avec celle des troupeaux du $\mathrm{XX}^{\mathrm{e}}$ siècle («En moyenne, les troupeaux sont passés de 200 brebis à plus de 500, voire 1000 à 3000 de nos jours »). À l'inverse du texte $\mathrm{A}$, les auteurs distinguent une activité pastorale ancienne et révolue d'une activité pastorale actuelle dont la relation de complémentarité avec la biodiversité serait rompue. Les dégradations causées par les troupeaux sont énoncées par accumulation. Le topos ${ }^{9}$ de l'excès qui y est associé - « surpâturage », «piétinement», « concentrations excessives »- produit une vision négative des interactions entre l'agropastoralisme moderne et l'environnement. Au-delà de l'activité pastorale, c'est le rapport de l'homme au territoire qui est condamné. La focalisation sur les facteurs humains à l'origine des difficultés rencontrées dans l'élevage rompt le lien de causalité entre loup et crise du pastoralisme ${ }^{10}$. La responsabilité du loup est désengagée, au profit de celle de l'homme : « Les problèmes des éleveurs datent d'avant l'arrivée du loup ", " sans que le loup y soit pour quelque chose ». Une nouvelle relation de causalité est ainsi instaurée : ce n'est plus le loup qui se trouve être la cause de la crise du pastoralisme, ce sont les activités humaines.

L'élevage, envisagé selon son rôle pour la sauvegarde d'une biodiversité à la fois sauvage et domestique, est légitimé, dans le texte $\mathrm{A}$, par un consensus humain : «pour qui apprécie », « appréciés par nos sociétés urbanisées », « nos paysages emblématiques ». La nature de ce consensus témoigne d'une vision dans laquelle l'appréciation culturelle du paysage fait autorité. En incarnant « l'une de nos agricultures les plus respectueuses de la biodiversité, reconnue en outre comme productrice d'une variété de services écosystémiques ", l'élevage confère à l'homme, du point de vue de son rôle pour l'environnement, un statut proche de celui du loup : celui $\mathrm{d}^{\prime}$ " espèce clé de voûte ». Le rôle du loup dans la biodiversité est remis en question à travers un distinguo faisant apparaître un paradoxe entre son rôle dans les milieux naturels du « Grand Nord américain comme eurasien » et son rôle en France, où il représenterait une menace pour l'agropastoralisme. Une opposition émerge alors entre la place souhaitée du loup - celle d'une espèce clé de voûte - et sa place en France, « où la géographie et l'histoire sont bien différentes ». L'atteinte portée à l'élevage par le loup devient, par voie de conséquence, une atteinte à la biodiversité dont la sauvegarde

9 Issu de la rhétorique classique, le topos renvoie, dans l'argumentation, au lieu commun. Le topos de l'excès peut se formuler ainsi : quand une chose est excessive, c'est une bonne raison pour la rejeter.

10 «Les problèmes des éleveurs [...] résultent plutôt de la forte concurrence internationale et de la baisse de consommation de viande ovine en France » ; « Le principal facteur [...] de disparition des terres agricoles en France est très nettement l'urbanisation. » est permise par le pastoralisme. Le parallèle entre apport du loup et apport de l'élevage pour la biodiversité rend possible l'instauration d'une relation concurrentielle entre une conception « sauvage » de la nature, où le loup serait une espèce " clé de voûte ", et une conception plus "domestiquée » de la nature, où le pastoralisme serait " producteur de services écosystémiques ». Le loup (dont l'intégration ne semble pas envisageable) mettrait alors en danger non le "modèle social français ", mais une sorte de «modèle naturel français ».

Le texte $\mathrm{B}$ s'oppose à cette deuxième conception de la biodiversité de façon explicite, en posant la question rhétorique : «Mais la nature domestiquée est-elle l'idéal de la biodiversité ? ». Les auteurs interrogent la notion même de biodiversité, segmentée, dans le texte $\mathrm{A}$, entre le sauvage et le domestique. La focalisation de ce dernier article sur les milieux ouverts est remise en cause, dans le paragraphe 3 du texte $B$, par une valorisation des milieux fermés, qui opère un recadrage de la notion de biodiversité. Ce recadrage conduit à la réaffirmation de la place du loup en France et à l'échelle internationale. Les auteurs du texte $B$ effacent les frontières dressées dans le texte A entre les effets du loup en France et «dans le Grand Nord américain comme eurasien », et positionnent, dans le paragraphe 6 , l'espèce " au sommet des réseaux trophiques ». Les rapports entre l'espèce et l'environnement étaient jusqu'ici essentiellement envisagés d'un point de vue écologique. Les liens existant entre le loup et les éleveurs sont caractérisés par des données réglementaires destinées à venir en aide au berger (« les aides à la prévention, les dispositifs de protection, $[\ldots]$ les indemnisations du bétail tué par les loups ») et qui, par leurs conséquences positives pour l'homme, viennent servir, dans l'argumentation, la cause du grand carnivore. Grâce à la réglementation en place, l'espèce devient un adjuvant du berger. $S^{\prime}$ il « détient la palme des effets en cascade positifs sur la biodiversité », le loup est également à l'origine $\mathrm{d}^{\prime}$ impacts positifs sur l'économie de la profession d'éleveur. Annoncé « fortement polémique, mais inévitable ", l'argument pragmatique par les conséquences positives ${ }^{11}$ évoque, mais très ironiquement, l'existence d'une communauté socioécologique dans laquelle le loup, en contribuant à l'ensemble de la biodiversité et à la pérennité des activités humaines, devient une espèce clé de voûte d'un environnement intégrant l'homme et la biodiversité. Parce qu'elle est polémique, l'évocation d'une telle communauté empêche d'envisager la possibilité même de la formation d'une

\footnotetext{
${ }^{11}$ L'argument pragmatique permet « d'appréhender un acte ou un événement en fonction de ses conséquences favorables ou défavorables » (Perelman et Olbrechts-Tyteca, 1958, p. 358). Ici, l'argument pragmatique consiste à présenter les conséquences positives, sur l'économie de la profession d'éleveur, des attaques des troupeaux par les loups.
} 
communauté socioécologique durable comprenant les hommes et les loups (Mathevet et Bousquet, 2014).

Le rapport des acteurs de la controverse à l'espace et au temps rend compte d'une rupture entre la nature et la société. L'ensemble socioécologique constitué d'humains et de non-humains est divisé, il se construit alternativement à travers la mise à l'écart totale ou partielle d'un acteur : le loup dans le texte A, et l'éleveur « moderne » dans le texte $B$.

\section{La représentation des auteurs des tribunes}

Même si les deux articles se caractérisent par un discours très majoritairement non marqué énonciativement ${ }^{12}$, les marques ponctuelles de la personne («je», «tu », « nous », « vous ») donnent lieu à la formation de deux autorités distinctes, respectivement d'ordre scientifique et militant.

Le péritexte permet d'identifier, en début et en fin de texte, les signataires des articles. Bien que la structure formelle de présentation des auteurs soit similaire pour les deux textes, son contenu varie et conduit, dans son rapport avec le texte, à une autorité discursive différente. Tout d'abord désignés par des expressions qui renvoient à un ensemble : «Un groupe de scientifiques » (texte A), et «Collectif» (texte B), chaque signataire est ensuite nommé en fin d'article (34 signataires pour le A, 7 pour le $\mathrm{B}^{13}$ ). L'expression «Un groupe de scientifiques» donne d'emblée une place centrale au statut des auteurs, alors que le nom « Collectif » met davantage en évidence la cohésion d'un ensemble d'individus défendant une cause.

Dans le texte A, l'inclusion des signataires à la sphère scientifique est renforcée par la mention, en fin d'article, de la profession et du rattachement institutionnel de chacun $\mathrm{d}^{\prime} \mathrm{eux}^{14}$. Ces précisions contribuent à légitimer les données juridiques, sociologiques ou encore écologiques avancées dans l'article. Dans le paragraphe 2, les données juridiques ( «les loups y sont une espèce strictement

\footnotetext{
12 Le « Plaidoyer » comporte six occurrences de la quatrième personne («nous », « notre »), pour un texte de 993 mots. « Cessons de diaboliser le loup ! » comporte quatre occurrences de la quatrième personne, pour un texte de 1008 mots.

13 Sur le site de Ferus «première association nationale pour la conservation de l'ours, du loup \& du lynx en France », une pétition intitulée «Plaidoyer pour des écosystèmes non désertés par les loups » est publiée le 16 décembre 2014. Elle est présentée comme l'article paru dans Le Monde. La pétition est cependant plus longue, et comporte un nombre de signataires beaucoup plus important (39).

${ }^{14}$ La liste compte majoritairement des chercheurs (28). Elle comprend également des personnes issues de milieux professionnels liés à l'environnement (6).
}

protégée ») et scientifiques (" "espèce clé de voûte" », "fonction de "régulateur" », " productrice d'une variété de services écosystémiques ») sont énoncées à la forme passive. Le responsable des propos n'est pas mentionné et les verbes introducteurs du discours rapporté témoignent d'une distance des signataires vis-à-vis des propos énoncés : «Jugés en péril en Europe » ; « ils sont considérés comme "espèce clé de voûte" ». En revanche, le participe passé « reconnu » («l'élevage pastoral, l'une de nos agricultures [...] reconnue en outre comme productrice d'une variété de services écosystémiques ») témoigne d'une plus forte appropriation, de la part des auteurs, du discours autre. Représentants des thématiques qu'ils abordent, les signataires apparaissent à même d'en évaluer les conséquences et la valeur. L'expertise scientifique qu'ils incarnent atténue la dimension subjective du vocabulaire et authentifie le discours. C'est aussi le cas pour les expressions « ce qui est considérable », " plus préoccupant », où la dimension évaluative, qui traduit une prise de position, est objectivée par le statut des auteurs.

À la différence du «Plaidoyer », le texte B fait apparaître en fin d'article le statut professionnel des signataires, mais également leur appartenance au domaine naturaliste ${ }^{15}$. Le rapport qu'ils entretiennent avec la nature contribue à la formation de l'ethos du «Collectif », et entre en interaction avec une argumentation pragmatique par les conséquences négatives, qui dresse une critique du rapport de l'homme à son environnement. À travers un vocabulaire relevant de la nature et $\mathrm{du}$ monde animal ${ }^{16}$, l'argumentation se focalise sur l'importance des équilibres naturels, que les activités humaines menacent.

L'identité explicitée des auteurs, en lien avec le contenu de leur argumentaire, rend compte de deux formes de légitimation du discours. Le texte A privilégie une approche "scientifique », alors que le texte B construit un argumentaire en lien avec une autorité militante en mettant l'accent sur la notion de « collectif », sur les implications naturalistes ou encore sur l'engagement des auteurs pour la protection de la nature.

Bien que l'argumentation développée par les signataires des deux articles privilégie l'effacement énonciatif $^{17}$, les usages ponctuels de la quatrième personne

\footnotetext{
15 Quatre des sept signataires sont associés à l'univers naturaliste (sur ces quatre, trois sont impliqués dans des structures de protection de la nature). Trois signataires appartiennent à la sphère scientifique, et deux à la sphère journalistique.

16 « insectes pollinisateurs et autres invertébrés », « criquets », « sauterelles », «faune sauvage », « zones humides », « forêt », " forêts matures », " petits carnivores »...

17 Les textes A et B sont majoritairement caractérisés par une absence de marques de la personne («je », «tu», « nous», « vous »).
} 
(«nous », «notre ») rendent possible, dans les deux documents, la désignation d'une communauté renvoyant soit aux seuls signataires («nous » exclusif), soit à un ensemble plus vaste composé des signataires et des lecteurs (« nous » inclusif). La formation d'une telle communauté élargie favorise l'implication du lecteur dans le discours.

Dans le texte A, le pronom «nous » n'apparaît pas, alors qu'il est utilisé à deux reprises dans le texte B. Dans cet article, les occurrences de "nous » sont associées à des verbes de prise de position (« souhaiter » et « affirmer ») et témoignent ainsi de l'explicitation, de la part des signataires, de leur responsabilité énonciative lorsque la thèse qu'ils défendent est réaffirmée ${ }^{18}$. Le collectif engage ainsi sa responsabilité, en défendant un point de vue. La dimension militante du groupe de signataires est alors explicite. Elle est également perceptible à la lecture du titre "Cessons de diaboliser le loup ! », qui implique le lecteur par l'emploi de l'impératif "Cessons ». Cette implication est injonctive, et conduit le lecteur à devoir adopter la position militante des auteurs, à s'intégrer au « collectif ». Dans le texte A, les marques de la quatrième personne (uniquement celles du possessif « notre », toujours inclusif) désignent tout au long du texte une communauté comprenant à la fois les signataires et leurs lecteurs. Associé aux noms «paysages », «agricultures", «pays » et « sociétés », le possessif témoigne d'une appropriation, par la communauté, d'éléments liés à la nature, sur lesquels l'homme porte une action («paysages », « agriculture »), et d'éléments liés au social (« société », «pays»). La communauté représentée par "notre » est caractérisée par son rapport à des éléments sur lesquels l'homme a une action. Le lecteur est, de fait, inclus dans cette communauté dont l'existence n'est pas discutée. Elle est posée en amont de l'argumentation. Le possessif « notre " apparaît majoritairement dans des groupes nominaux thématiques, et renvoie ainsi à ce qui est présupposé ou préconstruit, ce qui est déjà connu et admis par le lecteur. De cette façon, envisager l'environnement en termes d'appropriation et de possession ne fait pas débat, et s'institue en connaissance partagée.

L'environnement dont il est question n'est autre que le territoire français. Déterminé par le possessif « notre », le substantif " pays » (" Inciter la grande faune à conserver un comportement sauvage dans nos pays exige une régulation attentive, vigoureuse parfois »; " Or, notre pays n'est pas le Wyoming ou le Montana ») désigne un espace à la fois naturel et culturel dans lequel l'homme a pleinement sa place, puisque cet espace lui appartient.

\footnotetext{
18 « Nous souhaitons vivement une approche responsable et éthique, loin des démagogies coutumières »; " nous affirmons, après bien d'autres, que le loup joue un rôle essentiel dans certains processus-clés des écosystèmes. »
}

L'intégration - par définition - de l'homme au territoire français contribue à rendre ce dernier responsable de ce $\mathrm{qu}^{\prime} \mathrm{il}$ advient de son environnement. Dans le paragraphe 8 , la mention de l'engagement de « la France [...] auprès de l'Unesco à préserver les paysages culturels de l'agropastoralisme » implique, par continuité, l'engagement de la communauté désignée par «notre ». La responsabilisation du lecteur se fait par l'engagement de son pays pour l'agropastoralisme. La communauté à laquelle renvoie «notre » se caractérise par la notion de territorialité. Dans la proposition «Inciter la grande faune à conserver un comportement sauvage dans nos pays exige une régulation attentive, vigoureuse parfois ", il est bien question d'un rapport de possession à des environnements (qui se limitent à la France et aux pays qui lui sont similaires) mais également du fait que, dans ces espaces, la faune sauvage doit être gérée par l'homme. C'est de cette gestion qu'il est question ici. L'humain apparaît possesseur, par définition, des éléments de la nature.

Dans le texte B, l'image du territoire français est également très présente (six occurrences du nom propre « France »), mais, à la différence du texte précédent, le pays n'est pas envisagé selon un rapport d'appropriation par l'homme. La France désigne tout d'abord le territoire du loup, premier occupant, ensuite chassé par l'homme (« revenus naturellement en France en 1992. Soixante-dix ans après avoir été éradiqués d'un territoire qu'ils habitent depuis des centaines de milliers d'années »). Les occurrences répétées du nom propre «France » ancrent la problématique de l'élevage et du loup sur le territoire national, et conduisent à justifier la nécessité d'une politique de gestion de l'espèce par le prestige et la grandeur du pays. La question rhétorique « Pourquoi la France ne pourrait-elle pas héberger [...] de loups quand nos voisins, l'Espagne et l'Italie, en tolèrent chacun 4 à 8 fois plus [...] ? » fait émerger une hiérarchie entre les différents pays, hiérarchie dans laquelle la France, si elle ne peut cohabiter avec l'espèce, trouve sa valeur diminuée (par rapport à celle des pays frontaliers). Dans la proposition « Peut-on sérieusement croire qu'un grand pays comme la France est incapable de trouver une solution? ?, le prestige accordé à la France (" un grand pays ») justifie d'envisager une cohabitation avec le loup. La valorisation de la France va de pair avec la défense d'une politique de protection du loup, dont la mise en place dépend des acteurs du territoire : " C'est une question de volonté ».

Si les signataires $\mathrm{du}$ « Plaidoyer » s'identifient à une communauté explicitée en discours par le possessif "notre », les groupes auxquels cette communauté s'oppose ne sont pas explicitement nommés. L'absence de désignation précise de la partie opposée atténue la dimension polémique du discours et participe de l'objectivation du propos. 
Le texte $B$, en revanche, nomme ses détracteurs, et acquiert en cela un caractère d'emblée polémique, puisqu'il oppose son argumentaire à celui d'un groupe explicitement identifié. Dès le premier paragraphe, « syndicaliste paysan », « maire » et « spécialistes » sont catégorisés comme des opposants au loup, à travers un argument par l'exemple. Les propos rapportés entre guillemets ${ }^{19}$ font mention des expressions du texte A et conduisent à associer les « spécialistes » (« même les spécialistes s'y mettent ») au « groupe de scientifiques » du premier article. L'axiologie négative que nous observons en cotexte du discours rapporté est présente tout au long $\mathrm{du}$ texte ${ }^{20}$. Elle renforce la dimension polémique de $1^{\prime}$ article $^{21}$. Le pronom impersonnel « on », qui désigne tantôt les signataires et leurs lecteurs et tantôt leurs adversaires, participe du ton polémique qui émane de l'article. Lorsqu'il renvoie aux signataires du «Plaidoyer », le pronom « on » construit une image plurielle de l'adversaire. Le " groupe de scientifiques » désigné par «on » est envisagé en tant que masse douée d'une intentionnalité ("très opportunément », «tenter ", « passer sous silence »). Cette masse est celle à laquelle se heurte le «Collectif ». Malgré des analogies formelles et énonciatives (l'effacement énonciatif notamment), ces deux textes incarnent des positionnements discursifs respectivement scientifique et militant, qui se construisent à travers les liens du texte et du péritexte, et au sein même du texte. Les marques de la personne ainsi que le vocabulaire utilisé dans chacun des deux articles (dominance ou non d'un vocabulaire axiologique négatif) conduisent à fonder une argumentation qui, pour le texte $\mathrm{A}$, se caractérise par une objectivité de forme et, pour le texte $\mathrm{B}$, par une subjectivité de forme.

\section{Conclusion}

L'analyse comparée des deux articles met en évidence des processus argumentatifs proches conduisant à des conclusions opposées. Les dimensions spatiales et temporelles mobilisées permettent d'exposer un équilibre entre nature et agropastoralisme dans le texte A, et entre loup et nature dans le texte $B$. Ces équilibres, respectivement rompus en raison du retour du loup et de l'élevage

\footnotetext{
19 «passionnés, inspirés par le respect du vivant», « intenable ».

20 «impacts négatifs », « détruits », « piétinement ou élimination », « contaminées », " pertes», « concentrations excessives », «facteurs négatifs », " pollution et compaction », "perte de cohésion », "paysages agropastoraux [...] se banalisent et s'artificialisent », " pression », « surexploitation », « disparition », « diaboliser », « négligerait », « refus », « si préoccupant », « démagogies coutumières ».

21 « de manière naïve », « mal suprême », « très opportunément».
}

«moderne », témoignent de la définition de communautés socioécologiques différentes. Dans le premier article, la forte opposition entre le loup et le pastoralisme conduit à considérer le loup comme une menace pour la biodiversité même, au point qu'il serait nécessaire de limiter drastiquement sa présence. Le second article situe l'humain dans un rapport destructeur à la nature, rapport auquel il serait possible de remédier, par l'accueil, symbolique, d'un grand prédateur. Chacun des deux articles construit un ethos contribuant à légitimer la thèse défendue. Dans le premier, nous avons pu observer que le statut des auteurs, en interaction avec l'argumentation, participait de l'objectivation formelle du texte, alors que dans le second cette interaction produit un discours davantage subjectif. Cette analyse montre comment la présence du loup est instaurée par les scientifiques impliqués dans le pastoralisme et la conservation de la nature et témoigne du rôle de l'autorité (scientifique et militante) dans la légitimation des positionnements.

Elle donne à voir comment les interactions entre monde domestique et monde sauvage révèlent via le loup un mode $\mathrm{d}^{\prime}$ interexistence brouillant les frontières et les repères habituels entre artificiel et naturel (Micoud et Bobbé, 2006), déstructurant les modes de vie et les pratiques spatiotemporelles progressivement établies au fil du temps et stabilisées ces dernières décennies. La redéfinition d'un monde commun, la possibilité de vivre ensemble sont rendues difficiles par les disqualifications ou les velléités de contrôle et de régulation tantôt du loup, tantôt des pratiques pastorales. Dans cet exercice de publicisation de la problématique du loup, ces articles participent d'une proposition morale quant au comportement que chacun doit avoir à l'égard du loup mais aussi des humains et des territoires de la biodiversité. Il ne s'agit pas ici de renvoyer les groupes d'acteurs dos à dos mais au contraire de les inviter à (re)nouer le dialogue, pour négocier leur interexistence (Mathevet, 2012), expliciter les interdépendances sociales et écologiques qui sous-tendent le conflit, mesurer les valeurs qui leur sont accordées.

\section{Références}

Akrich M., Callon M., Latour B., 2006. Sociologie de la traduction. Textes fondateurs, Paris, Presses de l'École des mines.

Benhammou F., 2007. Crier au loup pour avoir la peau de l'ours. Une géopolitique locale de l'environnement à travers la gestion et la conservation des grands prédateurs en France. Thèse de doctorat en géographie, Paris, AgroParisTech.

Blandin P., 2009. De la protection de la nature au pilotage de la biodiversité, Versailles, Quæ.

Breton P., 2009 [1 ${ }^{\mathrm{re}}$ éd. 1996]. L'argumentation dans la communication, Paris, La Découverte.

Doré A., 2011. Des loups dans la cité. Éléments d'écologie pragmatiste. Thèse de doctorat en sociologie, Paris/Liège, Institut d'études politiques/Université de Liège. 
Labov W., 1972. Some principles of linguistic methodology, Language in Society, 1, 1, 97-120.

Landry J.-M., 2006 [1 $1^{\text {re }}$ éd. 2001]. Le loup. Biologie, mours, mythologie, cohabitation, protection, Lausanne/Paris, Delachaux et Niestlé.

Latour B., 1999. Politiques de la nature. Comment faire entrer les sciences en démocratie?, Paris, La Découverte.

Lescureux N., Linnell D.J.C., 2013. The effect of rapid social changes during post-communist transition on perceptions of the human-wolf relationships in Macedonia and Kyrgyzstan, Pastoralism, 3, 4, 1-20.

Lits M., 2008. Le récit, du mythe aux médias de masse, in Lits M., Du récit au récit médiatique, Bruxelles, De Boeck, 7-47.

Maldidier D., 1993. L'inquiétude du discours. Un trajet dans l'histoire de l'analyse du discours: le travail de Michel Pêcheux, Semen, 8, 2-8, http:/ / semen.revues.org/4351.

Mathevet R., 2012. La solidarité écologique. Ce lien qui nous oblige, Arles, Actes Sud.

Mathevet R., Bousquet F., 2014. Résilience E environnement. Penser les changements socio-écologiques, Paris, BuchetChastel.

Mathevet R., Thompson J., Delanoë O., Cheylan M., GilFourrier C., Bonnin M., 2010. La solidarité écologique : un nouveau concept pour la gestion intégrée des parcs nationaux et des territoires, Natures Sciences Sociétés, 18, 4, 424-433.

Reçu le 2 décembre 2015. Accepté le 4 mai 2016.
Mauz I., 2006. Introductions, réintroductions: des convergences, par-delà les différences, Natures Sciences Sociétés, 14 , supplément, S3-S10.

Micoud A., Bobbé S., 2006. Une gestion durable des espèces animales est-elle possible avec des catégories naturalisées ?, Natures Sciences Sociétés, 14, supplément, S32-S35.

Moriceau J.-M., 2011. L'homme contre le loup. Une guerre de deux mille ans, Paris, Fayard.

Mounet C., 2007. Les territoires de l'imprévisible. Conflits, controverses et "vivre ensemble " autour de la gestion de la faune sauvage. Le cas du loup et du sanglier dans les Alpes françaises. Thèse de doctorat de géographie, Grenoble, Université Joseph-Fourier.

Perelman C., Olbrechts-Tyteca L., 1958. Traité de l'argumentation : la nouvelle rhétorique, Bruxelles, Éditions de l'Université de Bruxelles.

Plantin C., 1990. Essais sur l'argumentation. Introduction à l'étude linguistique de la parole argumentative, Paris, Kimé.

Toulmin S.E., 1958. The uses of argument, Cambridge, Cambridge University Press.

Uexküll J. von, 2004. Mondes animaux et monde humain, Paris, Pocket Ago. Traduit de : Streifzüge durch die umwelten von tieren und menschen, Berlin, Springer, 1934.

Waltereit R., 2003. Le rapport dépendanciel entre adjectif et nom: données syntaxiques et structures conceptuelles, Syntaxe et Sémantique, 1, 4, 179-194. 